IJASC 13-2-5

\title{
Thermal Characteristics of the Optimal Design on 20W COB LED Down Light Heat Sink
}

\author{
Jae-Hyun Kwon", Jun-Myung Lee*, Wei Huang"*, Keon-Jun Park*, and Yong-Kab Kim*"†
}

\author{
*Department of Information and Communication Engineering, \\ Wonkwang University, South Korea \\ \{kojman, junmyung, bird75, ykim\}@wonkwang.ac.kr \\ ${ }^{* *}$ School of Computer and Communication engineering, Tianjin University of Technology, Tianjin 300191, China \\ huangwabc@163.com
}

\begin{abstract}
As the demand of the LED for lighting that emits light by p-n junction is increasing, studies on heatproof plate technology is being conducted to minimize the temperature of the LED lighting. As for the temperature of the LED devices, their light emitting efficiency decreases and the maximum lifespan drops down to 1/5. Therefore there are heat dissipation studies going on to minimize the heat. For LED heat dissipation, aluminum heat sink plates are mostly used. For this paper, we designed heat sink that fits residential $20 \mathrm{~W}$ COB LED Down Light; packaged the heat sink and 20W COB and analyzed and evaluated the thermal properties through a Solidworks flow simulation. We are planning to design the optimal heat sink plate to solve the thermal agglomeration considering $\operatorname{TIM}($ Thermal Interface material).
\end{abstract}

Key words : Heat sink, Solidworks Simulation, LED, Junction Temperature, Optimum Design

\section{INTRODUCTION}

Due to the increased efficiency and decreased price of the current Light emitting diode (LED) semiconductor, the use of lighting fixtures is growing. The LED lighting fixtures developed for study has been commercialized solving the heat dissipation problem, but as the social demand for high capacity increases, the single heat capacity of semiconductor element is getting larger and various ways to solve heat problems have been proposed. When converting electrical energy into light energy, heat is generated. Such heat and LED's heat dissipation are important factors to consider when improving the operation of LED and its reliability [1-2].

In general, high power Chip on Board (COB) LED lighting uses the heat sink made of aluminum with

Manuscript received: Sept. 26, 2013 / revised : Nov. 20, 2013

Corresponding Author: ykim@wonkwang.ac.kr

Tel: +82-063-850-6695, Fax: +82-063-850-6074.

Dept. of Information and Communication Eng., Wonkwang Univ., Korea insulation area expansion pins installed in it as a cool system dissipating heat on PCB. [3-4]. In order to develop a cooling system, the target amount of dissipation, the environment and possible temperature must be set; and the prediction of heat transfer coefficient and the design of the cooling system should be made[5-6]. In this paper, we will design a heat sink plate for 20W COB LED down light and look at the thermal properties through a Solidworks Flow simulation.

\section{20W COB LED DOWN LIGHT HEAT SINK}

In Section 1, we will describe the characteristics of LED heat dissipation and basic steps of heat dissipation design. In Section 2, we describe the structure of the COB package (pkg). In section 3, we will deal with designing optimal COB LED dissipation and how temperature will change depending on the pin thickness in our experiments. In section 4, we look at designing Heat Sink plates for $20 \mathrm{~W}$ COB LED. 


\subsection{LED heat dissipation characteristics and basic design}

The main reason why LED heat is generated is involved with the semiconductor junction due to the P-N junction. The LED temperature can be predicted by measuring the temperature of the junction but it is hard to measure it directly. Therefore the junction temperature is usually predicted by measuring the forward voltage $\left(\mathrm{V}_{\mathrm{f}}\right)$ and the thermal resistance. In order to improve its performance while maintaining the rate of LED, the LED junction temperature must be reduced below the maximum rated temperature when switching on and off the light. The most effective dissipation design method to dissipate the LED heat must be identified to design the most optimal dissipater. Using the computer fluid dynamics (CFD), the distribution and flow of temperature of the target area should be predicted [7-10]. Figure 1 shows the basic structure of the COB and Secondary Heat Sink.

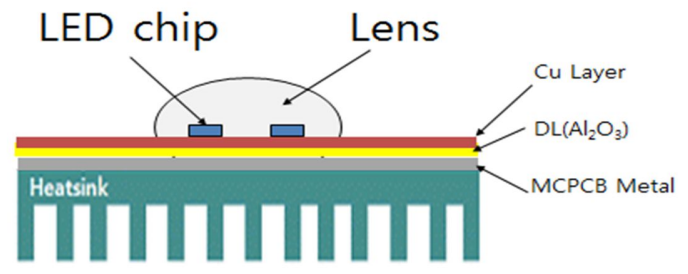

Figure 1. The basic structure of COB LED.

\subsection{Structure of COB-type LED Package}

In this paper, we used $20 \mathrm{~W}$ COB package as shown in Figure 2. The metal core printed circuit board (MCPCB) has the structure of arrays of $48 \mathrm{ea} 0.45 \mathrm{~W}$ chips. Heat problem is expected since it has a lot of chips on a small area. When designing the $\mathrm{COB}$ package, we designed in consideration of Copper Layer, Dielectric (DL) and MCPCB.
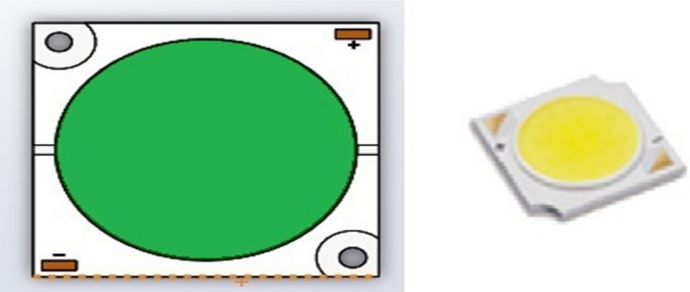

Figure 2. 20W COB Product and Simulation design.

\subsection{The optimal thermal design for COB LED}

The heat sink was designed to solve the heat problem of LED and 20W COB PKG that is optimal for residential purposes. As for the pin design, it is important to find the optimal pin thickness and the optimal pin-to-pin gap. If you design the pins without any gap in between in an attempt to expand the area, recycle symptoms could appear and the heat transmitted into the air could be passed into the inside through the dissipater. The experimental method sets the pin intervals of the 6061-based aluminum using Solidworks by changing the thickness of the pin and the number of pins at the same time.

Figure 3 shows how the temperature changes depending on how thick the pins are when the length of the pins of the dissipater designed by Solidworks and base are the same. The result of the experiments shows that when the pin is $2.5 \mathrm{~mm}$ thick, its temperature and air temperature remains low, meaning that heat dissipation is the most efficient.

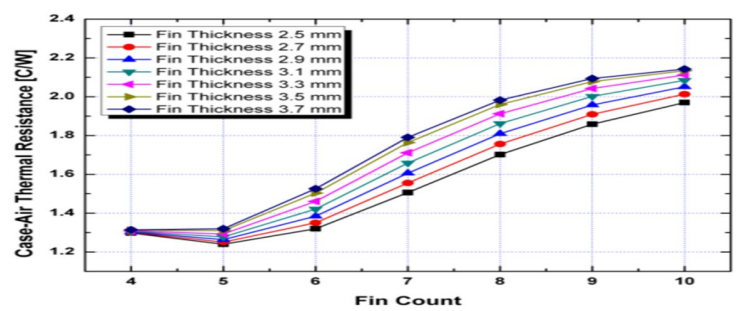

(a) Thermal resistance of the air.

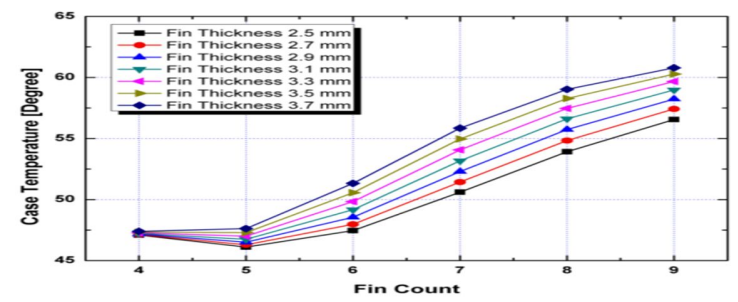

(b) Case Temperature.

Figure 3. Temperature change depending on how thick the COB LED pins.

\subsection{W COB LED Heat sink design and experiment}

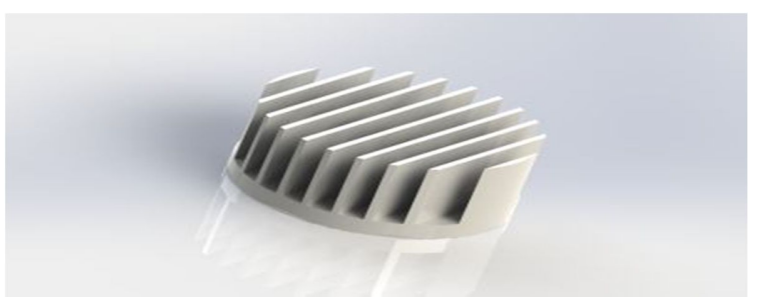

(a) Designed COB heat sink.

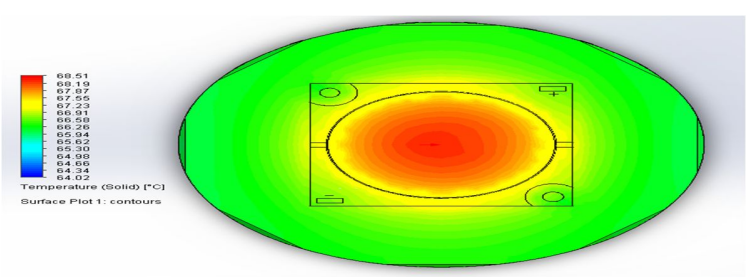

(b) The COB Package Simulation.

Figure 4. Designed COB LED heat sink and Simulation 
As shown in Figure 4, we designed 20W COB LED heat sink with condition set through Solidworks flow simulation at area of 1 , internal temperature of $25^{\circ} \mathrm{C}$, the heat transfer coefficient of $5 \mathrm{~W} / \mathrm{m}^{\circ} \mathrm{C}$, emissivity of 0.5 .

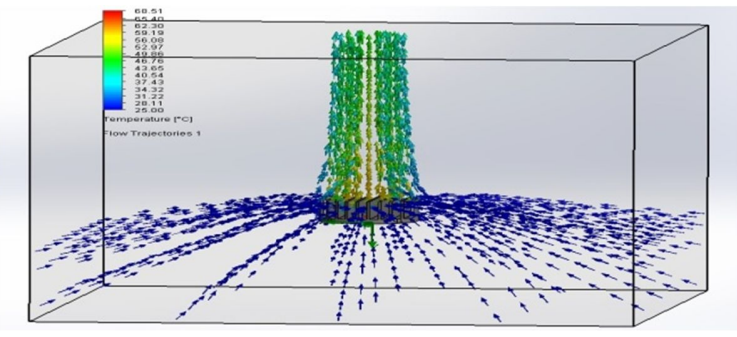

(a) Experimental results of air flow.

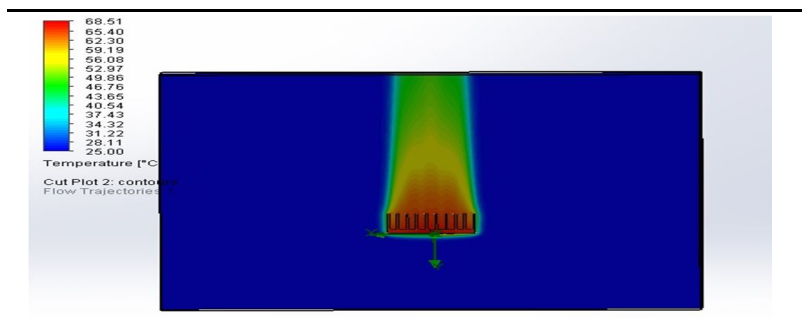

(b) Heat Distribution of COB and secondary Heat Sink. Figure 5. The results of thermal simulation.

As a result (Figure 5) of the simulation, the highest temperature was $68.51{ }^{\circ} \mathrm{C}$ with $4.5^{\circ} \mathrm{C}$ maximum difference in temperature. This result came from the condition where thermal interface material (TIM) was not included. To lower the temperature further, it is deemed that TIM should be considered.

\section{CONCLUSION AND FUTURE WORK}

LED must have long lifetime, maintain a constant brightness and colors for a certain period of time in order to be applied to various areas. The junction temperature must be lowered in order to solve these problems, thus solving the heat problem; and the heat must be efficiently dissipated through optimal heat dissipation design. In this paper, we designed a COB LED module and experimented on how temperature changes according to the thickness of the pins. As a result, we found out the optimal thickness of the pin, which is $2.5 \mathrm{~mm}$.

Also we have designed 20W COB LED heat sink and analyzed the thermal properties. The maximum temperature of $68.51{ }^{\circ} \mathrm{C}$ was relatively high. TIM was excluded. We are planning to design an optimal heat sink taking optimal heat sink and TIM and evaluate and demonstrate the performance.

\section{ACKNOWLEDGMENT}

This research was supported by Basic Science Research Program through the National Research Foundation of Korea (NRF) funded by the Ministry of Education (2013R1A1A2011835).

\section{REFERENCES}

[1] G.B. Hong, T.S Jang and Y.K. Kim, "Analysis of LED visible light communications system based on remote dimming control," Korea Institute of Information and Communication Technology Electronics, Vol.4, No.3, pp 153-157, 2011.

[2] Eo Iksu, "Analysis of heat dissipation of the LED lighting fixtures using CF-design," Korea Academic Industrial Society, Vol 9, No.6, pp. 1565-1568, 2008.

[3] Y.L. Lee and S.H. Hwang, "Study on Thermal Design of a 3W MR16 Light with single High-Power LED," J. of the KAICS, Vol. 11, No. 4, pp. 1023-1209. 2010.

[4] JEDEC Standard EIZ/JESD51-1, Integrated Circuits Thermal Measurement Method-Electrical Test Method (Single Semiconductor Device), EIA, 1955.

[5] K.H. Um and S.T Yoo "A Study for Designing of Intelligent Lighting Control LED Apparatus," The Journal of the Institute of Internet, Broadcasting and Communication(JIIBC), Vol.8,No.5, pp. 221-227, 2008.

[6] F. Farahmand, F. P. Dawson and J. D. Lavers "Temperature Rise and Free Convection Heat Transfer Coefficient for 2-D Pot-Core Inductors and Transformers," Institute of Electrical and Electronics Engineers(IEEE), pp. 2622-2629, 2005.

[7] T.S. Jung and H.K. Kang, "Investigation of Natural Convective Heat Flow Characteristics of Heat Sink," Korean Soc. Mech. Eng. Vol. 37, No. 1, pp. 27-33, 2013.

[8] B.G Kim, K.W Kim, T.S Jang, J.M Lee and Y.K Kim, "Development of Current Control System for solar LED street Light System," Journal of Advanced Smart Convergence(JASC), Vol1, No1, pp52-56, 2012.

[9] J.W Lee and J.W Im, " A Study on the Development of LED Spot Luminaire for Image Lighting," The Institute of Internet, Broadcasting and Communication(IIBC), Vol.10, No.5, pp. 271-276, 2010.

[10] J.M Han and D.S Seo, "Electro-optical Characteristics of LED Flat Light Source in Low Temperature Condition," The Institute of Internet, Broadcasting and Communication(IIBC), Vol.11, No.1, pp.61-65, 2011.

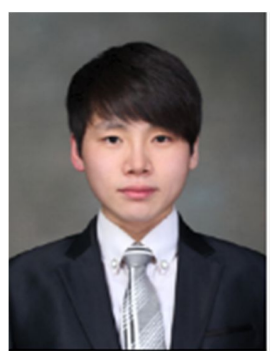

Jae Hyun Kwon received the B.S degree in Electrical Information Communication Engineering from Wonkwang University. He is currently the master's course in Information Comm. Engineering, Wonkwang University, Korea. His research interests are LED display, Heat-sink and Optical visible communication. 


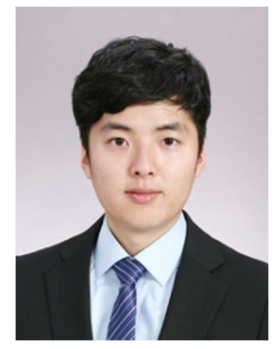

Jun Myung Lee received the B.S degree in Electrical Information Communication Engineering from Wonkwang University. He is currently the master's course in Information Comm. Engineering, Wonkwang University, Korea. His research interests are LED display, Heat-sink, Optical visible communication and Photodiode.

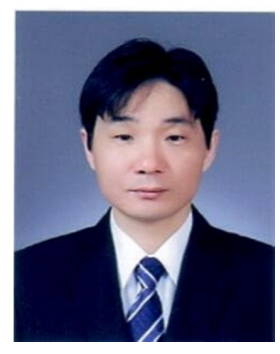

Keon Jun Park received the B.S degree in Electrical \& Electronics Engineering from the Wonkwang University, Iksan, South Korea, in 2003, and the M.S degree in Control and Instrumentation Engineering in 2005. He received the $\mathrm{Ph} . \mathrm{D}$ degree in Electrical Engineering from University of Suwon, Suwon, South Korea, in 2010. He is currently a research professor with Information and Communication Engineering, Wonkwang University, Korea. His research interests include computational intelligence, intelligence modeling and control, and pattern recognition.

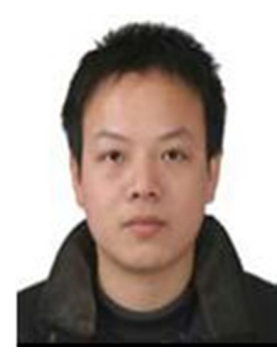

Wei Huang received the MS degree at school of information Engineering, East China Institute of Technology, China, in 2006, and Ph.D. degree at State Key Laboratory of Software Engineering, Wuhan University, China, in 2011. He is currently a lecturer in the School of Computer and Communication Engineering, Tianjin University of Technology, Tianjin, China. His research interests include evolutionary computation, operations research, fuzzy system, natural language processing, and software reliability.

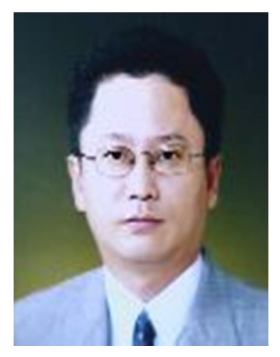

Yong Kab Kim received the Ph.D degree in Electrical \& Computer Engineering from North Carolina State University. He is currently in Professor in Dep't of Electrical and Information Comm. Engineering, Wonkwang University, Korea. He is a director of Wonkwang University Business Incubator and LED Research Center. His research interests are remote sensing for visible communication, LED communication, optical fiber sensing, Power intelligent control, and Fuzzy sensing control. 